\title{
On Norm Attaining Polynomials
}

\author{
By \\ Richard M. Aron, Domingo García** and Manuel Maestre***
}

\begin{abstract}
We show that for every Banach space $X$ the set of 2-homogeneous continuous polynomials whose canonical extension to $X^{* *}$ attain their norm is a dense subset of the space of all 2-homogeneous continuous polynomials $\mathcal{P}\left({ }^{2} X\right)$.
\end{abstract}

\section{$\S 1 . \quad$ Introduction}

This note continues recent work on generalizations of the Bishop-Phelps theorem [6], on the density of the set of norm attaining continuous linear functionals on a Banach space $X$ in $X^{*}$. The most significant such extension was done nearly 40 years ago by Lindenstrauss [13], who gave examples of Banach spaces $X$ and $Y$ for which the set of norm attaining linear operators is not a dense subset of $\mathcal{L}(X, Y)$. He also proved that the set of continuous linear operators $T \in \mathcal{L}(X, Y)$ between Banach spaces $X$ and $Y$ whose second transpose $T^{* *}$ attains its norm on $X^{* *}$ is dense in $\mathcal{L}(X, Y)$.

Using the obvious isometry between $\mathcal{L}\left(X, Y^{*}\right)$ and the space $\mathcal{L}\left({ }^{2}(X \times Y)\right)$ of continuous bilinear forms on $X \times Y$, one can ask questions about density of

Communicated by T. Kawai. Received August 23, 2001. Revised January 15, 2002. 2000 Mathematics Subject Classification(s): Primary 46B28; Secondary 46B04.

Key words: Norm attaining operators, polynomials.

The first author is supported by a grant of the Ministerio de Educación y Cultura of Spain (SAB1999-0214), the second and third authors are supported by DGES (Spain) PB. 96-0758.

* Department of Mathematical Sciences, Kent State University, Kent, Ohio 44242, USA. e-mail: aron@mcs.kent.edu.

** Departamento de Análisis Matemático, Universidad de Valencia, 46100 Burjasot (Valencia), Spain. e-mail: domingo.garcia@uv.es

*** Departamento de Análisis Matemático, Universidad de Valencia, 46100 Burjasot (Valencia), Spain.

e-mail: maestre@uv.es 
norm-attaining bilinear forms. In fact, in the most common case $X=Y$, there are three sets of natural questions which can be asked:

(i) a) Is the set of norm attaining bilinear forms on $X$ dense in $\mathcal{L}\left({ }^{2}(X \times X)\right)=$ $\mathcal{L}\left({ }^{2} X\right) ?$

b) Is the set of bilinear forms, whose extension to $X^{* *} \times X^{* *}$ is norm attaining, dense in $\mathcal{L}\left({ }^{2}(X \times X)\right)$ ?

(ii) a) Is the set of norm attaining symmetric bilinear forms $X \times X \rightarrow \mathbb{K}$ dense in the space $\mathcal{L}_{s}(2(X \times X))$ of symmetric bilinear forms?

b) Is the set of symmetric bilinear forms, whose extension to $X^{* *} \times X^{* *}$ is norm attaining, dense in $\mathcal{L}_{s}\left({ }^{2}(X \times X)\right)$ ?

(iii) a) Is the set of norm attaining 2-homogeneous polynomials $X \rightarrow \mathbb{K}$ dense in the space of 2 -homogeneous polynomials $\mathcal{P}\left({ }^{2} X\right)$ ?

b) Is the set of 2-homogeneous polynomials whose canonical extension to $X^{* *}$ is norm attaining dense in $\mathcal{P}\left({ }^{2} X\right)$ ?

(We recall that $\mathcal{P}\left({ }^{n} X\right)$ denotes the Banach space of $n$-homogeneous continuous polynomials $P: X \rightarrow \mathbb{K}$, and that, by definition, each such $P$ is associated with a unique continuous symmetric $n$-linear form $A: X \times \cdots \times X \rightarrow \mathbb{K}$ by $P(x)=A(x, \ldots, x)$. The norm of $P$ is $\|P\|:=\sup \{|P(x)|:\|x\| \leq 1\}$, the norm of an element $A$ in the space $\mathcal{L}\left({ }^{n} X\right)$ of continuous $n$-linear forms is $\|A\|:=\sup \left\{\left|A\left(x_{1}, \ldots, x_{n}\right)\right|:\left\|x_{j}\right\| \leq 1, j=1, \ldots, n\right\}$, and the relation between the norm of an $n$-homogeneous polynomial and that of the associated symmetric $n$-linear form is $\|P\| \leq\|A\| \leq\left(n^{n} / n !\right)\|P\|$. See [10] for background information.)

These questions are perhaps a bit more subtle than may first appear. For example, the fact that for $T \in \mathcal{L}\left(X, Y^{*}\right)$, there is a point $x_{0} \in X,\left\|x_{0}\right\|=1$, such that $\|T\|=\left\|T\left(x_{0}\right)\right\|$ does not imply that there are vectors $x_{1}, y_{1}$ of norm 1 such that for the corresponding bilinear form $A \in \mathcal{L}\left({ }^{2}(X \times Y)\right),\|T\|=\|A\|=$ $A\left(x_{1}, y_{1}\right)$.

There are partial positive answers to part a) of each of the three questions. For example, Finet, Werner, and the first author showed in [5] that if $X$ has the Radon-Nikodym property, then the set of norm attaining $n$-linear forms on $X$ is dense in $\mathcal{L}\left({ }^{n} X\right)$ for every $n \geq 1$. In [8], Choi and Kim obtained positive results for part a) of all three questions when, for example, $X$ has the DunfordPettis property with shrinking basis. In general, however, part a) of the three questions has a negative answer. In [2], Acosta, Aguirre, and Payá showed that 
the answer to the first part of questions (i) and (ii) is negative for $X=G$, the Gowers space. An independent example was given by Choi [7] for $X=L_{1}[0,1]$. Nevertheless, every linear operator $L_{1}[0,1] \rightarrow L_{\infty}[0,1]$ can be approximated by a norm attaining linear mapping ([11]). Moreover, Jiménez and Payá showed that the set $N A\left(\mathcal{P}\left({ }^{n} X\right)\right)$ of norm attaining $n$-homogeneous polynomials on a Banach space $X$ is not norm dense in the Banach space $\mathcal{P}\left({ }^{n} X\right)$ of all $n$ homogeneous polynomials on $X$ ([12, Theorem 3.2], see also [2]).

The situation for part b) of these questions is quite different. In 1998 Acosta proved in [1, Theorem 1] that the subset of $\mathcal{L}\left({ }^{2} X\right)$, consisting of all bilinear forms on $X$ whose third Arens transpose attains its norm, is dense in $\mathcal{L}^{2}(X)$. In this note, we answer part b) of question (iii), by proving that for every Banach space $X$, the set of all 2-homogeneous polynomials whose canonical extension [4] to $X^{* *}$ attain their norm is a dense subset of $\mathcal{P}\left({ }^{2} X\right)$. We obtain this result by using a variation of Lindenstrauss' original argument. We also sharpen Acosta's result. The authors are unaware of any progress on part b) of question (ii). In addition, the analogue of Lindenstrauss' result for $n$-homogeneous and $n$-linear forms when $n \geq 3$ remains open.

\section{$\S 2 . \quad$ Results}

For every $A \in \mathcal{L}\left({ }^{2}(X \times Y)\right)$ we have two possible extensions to $X^{* *} \times Y^{* *}$

$$
A_{12}\left(x^{* *}, y^{* *}\right):=\lim _{\alpha} \lim _{\beta} A\left(x_{\alpha}, y_{\beta}\right), \quad A_{21}\left(x^{* *}, y^{* *}\right):=\lim _{\beta} \lim _{\alpha} A\left(x_{\alpha}, y_{\beta}\right)
$$

where $\left(x_{\alpha}\right) \subset X$, resp. $\left(y_{\beta}\right) \subset Y$, are nets converging weak-* to $x^{* *}$, resp. $y^{* *}$. In general $A_{12} \neq A_{21}$ but $\left\|A_{12}\right\|=\|A\|=\left\|A_{21}\right\|$. It is very easy to check that $A^{t t t}=A_{12}$ where $A^{t t t}$ is the third Arens transpose of $A$ [3]. If $P$ is a 2-homogeneous polynomial on a Banach space $X$ and $A$ is the associated symmetric bilinear form, then the canonical extension of $P$ to the bidual $X^{* *}$ is given by $\tilde{P}\left(x^{* *}\right):=A_{12}\left(x^{* *}, x^{* *}\right)=A_{21}\left(x^{* *}, x^{* *}\right)$, for all $x^{* *} \in X^{* *}$. In [9] it is proved that $\|\tilde{P}\|=\|P\|$.

The following is the polynomial analogue of [1, Theorem 1].

Theorem 2.1. $\quad$ For every Banach space $X$, the set of all 2-homogeneous polynomials on $X$ whose extension to $X^{* *}$ is norm attaining is dense in $\mathcal{P}\left({ }^{2} X\right)$.

Proof. We will emphasize where our argument, which is based on that of $\left[13\right.$, Theorem 1], differs from it. Let $P \in \mathcal{P}\left({ }^{2} X\right)$ be such that $\|P\|=1$, let $A \in \mathcal{L}_{s}\left({ }^{2} X\right)$ be the symmetric bilinear mapping associated to $P$, and let $\epsilon$ with 
$0<\epsilon<1 / 3$ be given. We choose first a monotonically decreasing sequence $\left\{\epsilon_{k}\right\}$ of positive numbers which satisfies the following conditions:

(1) $8 \sum_{i=1}^{\infty} \epsilon_{i}<\epsilon, \quad 8 \sum_{i=k+1}^{\infty} \epsilon_{i}<\epsilon_{k}^{2}, \quad$ and $\epsilon_{k}<1 /(10 k), \quad k \in \mathbb{N}$.

Using induction, we next choose sequences $\left\{P_{k}\right\}_{k=1}^{\infty}$ in $\mathcal{P}\left({ }^{2} X\right)$ and $\left\{x_{k}\right\}_{k=1}^{\infty}$ in $X$, satisfying

(2) $\quad P_{1}=P$,

(3) $\quad P_{k}\left(x_{k}\right) \geq\left\|P_{k}\right\|-\epsilon_{k}^{2}, \quad$ and $\left\|x_{k}\right\|=1, \quad k \in \mathbb{N}$,

(4) $\quad P_{k+1}(x):=P_{k}(x)+\epsilon_{k} A_{k}\left(x, x_{k}\right)^{2}, \quad x \in X, \quad k \in \mathbb{N}$,

where $A_{k} \in \mathcal{L}_{s}\left({ }^{2} X\right)$ is the unique symmetric bilinear form associated to $P_{k}$.

Having chosen these sequences we see that the following hold.

$$
\left\|P_{j}-P_{k}\right\| \leq 4(4 / 3)^{2} \sum_{i=j}^{k-1} \epsilon_{i}, \quad\left\|P_{k}\right\| \leq 4 / 3, \quad j<k, \quad k \in \mathbb{N} .
$$

We prove this assertion by induction on $k$. Note that

$$
\begin{gathered}
\left\|P_{1}-P_{2}\right\|=\sup _{\|x\| \leq 1} \epsilon_{1}\left|A_{1}\left(x, x_{1}\right)\right|^{2} \leq \epsilon_{1}\left\|A_{1}\right\|^{2} \leq 4 \epsilon_{1}\left\|P_{1}\right\|^{2}=4 \epsilon_{1}<4(4 / 3)^{2} \epsilon_{1}, \text { and } \\
\left\|P_{2}\right\| \leq\left\|P_{1}\right\|+4 \epsilon_{1}<1+\frac{4}{24}<\frac{4}{3},
\end{gathered}
$$

so that (5) holds for $k=2$.

If we assume that (5) is true for a given $k \in \mathbb{N}$, we have that

$$
\left\|P_{k}-P_{k+1}\right\|=\sup _{\|x\| \leq 1} \epsilon_{k}\left|A_{k}\left(x, x_{k}\right)\right|^{2} \leq \epsilon_{k}\left\|A_{k}\right\|^{2} \leq 4 \epsilon_{k}\left\|P_{k}\right\|^{2}<4(4 / 3)^{2} \epsilon_{k},
$$

since $\left\|A_{k}\right\| \leq 2\left\|P_{k}\right\|$. Thus $\left\|P_{j}-P_{k+1}\right\| \leq\left\|P_{j}-P_{k}\right\|+\left\|P_{k}-P_{k+1}\right\|<$ $4(4 / 3)^{2} \sum_{i=j}^{k} \epsilon_{i}$ and $\left\|P_{k+1}\right\| \leq\left\|P_{1}\right\|+\left\|P_{k+1}-P_{1}\right\|<1+4(4 / 3)^{2} \sum_{i=1}^{k} \epsilon_{i}<$ $1+4(4 / 3)^{2}(1 / 8) \epsilon<1+(16 / 9)(1 / 6)<4 / 3$, because of $(1)$.

(6) $\left(\left\|P_{k}\right\|\right)$ is a strictly increasing sequence, $\left\|P_{k+1}\right\|>\left\|P_{k}\right\|+\epsilon_{k}\left\|P_{k}\right\|^{2}-4 \epsilon_{k}^{2}$, and $\left\|P_{k}\right\| \geq 1, \quad k \in \mathbb{N}$.

$$
\left|P_{j+1}\left(x_{k}\right)\right| \geq\left\|P_{j+1}\right\|-2 \epsilon_{j}^{2}, \quad j<k, \quad k \in \mathbb{N}
$$

The proof of these relations is very similar to that of the corresponding estimates in [13].

$$
\left|A_{j}\left(x_{k}, x_{j}\right)\right|^{2} \geq\left\|P_{j}\right\|^{2}-6 \epsilon_{j}, \quad j<k, \quad k \in \mathbb{N}
$$


By using (6) and (7) we get $\left\|P_{j}\right\|+\epsilon_{j}\left|A_{j}\left(x_{k}, x_{j}\right)\right|^{2} \geq\left|P_{j}\left(x_{k}\right)\right|+\epsilon_{j}\left|A_{j}\left(x_{k}, x_{j}\right)\right|^{2} \geq$ $\left|P_{j+1}\left(x_{k}\right)\right| \geq\left\|P_{j+1}\right\|-2 \epsilon_{j}^{2} \geq\left\|P_{j}\right\|+\epsilon_{j}\left\|P_{j}\right\|^{2}-6 \epsilon_{j}^{2}$. Hence $\epsilon_{j}\left|A_{j}\left(x_{k}, x_{j}\right)\right|^{2} \geq$ $\epsilon_{j}\left\|P_{j}\right\|^{2}-6 \epsilon_{j}^{2}$, and (8) follows.

Since $\left\|P_{j}-P_{k}\right\| \leq 8 \sum_{i=j}^{k-1} \epsilon_{i}$ and $\left\|A_{j}-A_{k}\right\| \leq 16 \sum_{i=j}^{k-1} \epsilon_{i}$, there exist elements $Q \in \mathcal{P}\left({ }^{2} X\right)$ and $B \in \mathcal{L}_{s}\left({ }^{2} X\right)$ which are the limits of these respective Cauchy sequences; moreover, it is clear that $B$ is the bilinear form which corresponds to $P$. Thus, given $\eta>0$, there exists $j_{0} \in \mathbb{N}$ such that $\left\|Q-P_{j}\right\| \leq\left\|B-A_{j}\right\|<\eta$ for all $j \geq j_{0}$. Hence $\left\|P_{j}\right\| \geq\|Q\|-\eta$ for all $j \geq j_{0}$. By using (8), we have $\left|B\left(x_{k}, x_{j}\right)\right| \geq\left|A_{j}\left(x_{k}, x_{j}\right)\right|-\left\|B-A_{j}\right\|>\sqrt{\left\|P_{j}\right\|^{2}-6 \epsilon_{j}}-\eta \geq$ $\sqrt{(\|Q\|-\eta)^{2}-6 \epsilon_{j}}-\eta$ for all $k>j \geq j_{0}, j, k \in \mathbb{N}$.

So, if $z \in X^{* *}$ is a cluster point of $\left(x_{k}\right)$ then

$$
\left|B_{21}\left(z, x_{j}\right)\right| \geq \sqrt{(\|Q\|-\eta)^{2}-6 \epsilon_{j}}-\eta
$$

for all $j \geq j_{0}, j \in \mathbb{N}$. Hence $\left|B_{21}(z, z)\right| \geq\|Q\|-2 \eta$, for all $\eta>0$. Therefore $|\tilde{Q}(z)|=\left|B_{21}(z, z)\right| \geq\|Q\|$, and $\|\tilde{Q}\|=|\tilde{Q}(z)|=\|Q\|$. This implies that $\tilde{Q}$ is norm attaining. On the other hand, by (1) and (5), we have $\left\|P-P_{k}\right\|=$ $\left\|P_{1}-P_{k}\right\| \leq 4(4 / 3)^{2} \sum_{i=1}^{k-1} \epsilon_{i}<8 \sum_{i=1}^{\infty} \epsilon_{i}<\epsilon$, for all $k \in \mathbb{N}$. Hence $\|P-Q\| \leq \epsilon$ and this concludes the proof.

The following is an improvement of M. Acosta's result [1, Theorem 1]. The proof, which is again a modification of Lindenstrauss' original argument, seems simpler to the authors than the one presented in [1]. The examples which follow show that this result does indeed have some content.

Theorem 2.2. The set of bilinear forms $A: X \times Y \rightarrow \mathbb{K}$ whose extensions $A_{12}$ and $A_{21}$ attain their norms simultaneously at the same points is dense in $\mathcal{L}\left({ }^{2}(X \times Y)\right)$.

Proof. Since the reasoning is very similar to that of [13] and Theorem 2.1 , we will give only a very brief sketch, indicating only where some (slight) differences appear.

Let $A \in \mathcal{L}\left({ }^{2}(X \times Y)\right)$ and $\epsilon, 0<\epsilon<1 / 3$, be given. We assume that $\|A\|=1$ and we choose a monotonically decreasing sequence $\left\{\epsilon_{k}\right\}$ of positive numbers which satisfies the following conditions:

$$
2 \sum_{i=1}^{\infty} \epsilon_{i}<\epsilon, \quad 2 \sum_{i=k+1}^{\infty} \epsilon_{i}<\epsilon_{k}^{2}, \quad \text { and } \epsilon_{k}<1 / 10 k, \quad k \in \mathbb{N} .
$$


We next choose sequences $\left\{A_{k}\right\}_{k=1}^{\infty} \subset \mathcal{L}\left({ }^{2}(X \times Y)\right)$ and unit vectors $\left\{x_{k}\right\}_{k=1}^{\infty} \subset$ $X$ and $\left\{y_{k}\right\}_{k=1}^{\infty} \subset Y$, satisfying

$$
\begin{gathered}
A_{1}=A, A_{k}\left(x_{k}, y_{k}\right) \geq\left\|A_{k}\right\|-\epsilon_{k}^{2}, \quad \text { and } \\
A_{k+1}(x, y):=A_{k}(x, y)+\epsilon_{k} A_{k}\left(x, y_{k}\right) A_{k}\left(x_{k}, y\right), \quad x \in X, y \in Y, \quad k \in \mathbb{N} .
\end{gathered}
$$

Arguing as above, $1 \leq\left\|A_{j}\right\| \leq\left\|A_{j+1}\right\| \leq 4 / 3$ for all $j$, and

$$
\epsilon_{j}\left|A_{j}\left(x_{k}, y_{j}\right) A_{j}\left(x_{j}, y_{k}\right)\right| \geq \epsilon_{j}\left\|A_{j}\right\|^{2}-6 \epsilon_{j}^{2} \quad \text { for all } j<k .
$$

The last inequality is a consequence of the following:

$$
\begin{aligned}
\left\|A_{j}\right\|+\epsilon_{j}\left|A_{j}\left(x_{k}, y_{j}\right) A_{j}\left(x_{j}, y_{k}\right)\right| & \geq\left|A_{j+1}\left(x_{k}, y_{k}\right)\right| \\
& \geq\left\|A_{j+1}\right\|-2 \epsilon_{j}^{2} \geq\left\|A_{j}\right\|+\epsilon_{j}\left\|A_{j}\right\|^{2}-6 \epsilon_{j}^{2} .
\end{aligned}
$$

Thus, $\epsilon_{j}\left|A_{j}\left(x_{k}, y_{j}\right)\right|\left\|A_{j}\right\| \geq \epsilon_{j}\left|A_{j}\left(x_{k}, y_{j}\right) A_{j}\left(x_{j}, y_{k}\right)\right| \geq \epsilon_{j}\left\|A_{j}\right\|^{2}-6 \epsilon_{j}^{2}$, from which it follows that

(1) $\left|A_{j}\left(x_{k}, y_{j}\right)\right| \geq\left\|A_{j}\right\|-6 \epsilon_{j}$, and by a similar argument, $\left|A_{j}\left(x_{j}, y_{k}\right)\right| \geq$ $\left\|A_{j}\right\|-6 \epsilon_{j}$ for all $j<k$.

The sequence $\left(A_{j}\right)$ converges in norm to a bilinear form $B$ satisfying $\|A-B\| \leq$ $\epsilon$. By (1) we will have

(2) $\left|B\left(x_{k}, y_{j}\right)\right| \geq\|B\|-\frac{1}{j}$ and $\left|B\left(x_{j}, y_{k}\right)\right| \geq\|B\|-\frac{1}{j}$, for all $k>j$.

Now if $x_{0}^{* *} \in X^{* *}$ and $y_{0}^{* *} \in Y^{* *}$ are weak-* cluster points of $\left(x_{j}\right)$ and $\left(y_{j}\right)$ respectively, by (2) we will have

$$
\left|B_{21}\left(x_{0}^{* *}, y_{j}\right)\right| \geq\|B\|-\frac{1}{j} \text { and }\left|B_{12}\left(x_{j}, y_{0}^{* *}\right)\right| \geq\|B\|-\frac{1}{j}, \text { for all } j \in \mathbb{N} .
$$

Hence

$$
\|B\|=\left\|B_{21}\right\| \geq\left|B_{21}\left(x_{0}^{* *}, y_{0}^{* *}\right)\right| \geq\|B\|=\left\|B_{12}\right\| \geq\left|B_{12}\left(x_{0}^{* *}, y_{0}^{* *}\right)\right| \geq\|B\|,
$$

and the result follows.

The first place that one might look for an example that the two natural extensions of a bilinear form behave differently with respect to norm attainment is the non-Arens regular space $\ell_{1}$, and indeed it is here that we have the following examples. 
Example 1. Let $A: \ell_{1} \times \ell_{1} \rightarrow \mathbb{K}$ be defined by

$$
A(x, y)=\sum_{j=1}^{\infty} \frac{j}{j+1}\left(\sum_{k=1}^{j} x_{2 k-1}\right) y_{2 j}+\sum_{j=1}^{\infty} \frac{j}{j+1}\left(\sum_{k=1}^{j} y_{2 k-1}\right) x_{2 j} .
$$

Then $A \in \mathcal{L}_{s}\left({ }^{2} \ell_{1}\right), A$ is not norm attaining, and there are points $z, w \in$ $B_{\ell_{1} * *}$ such that $1=\|A\|=A_{12}(z, w)=A_{21}(w, z), A_{12}(w, z)=A_{21}(z, w)=0$.

We omit the details of proof for this example, since they are similar to those of Example 2, below.

Example 2. There is a bilinear form $A$ on $\ell_{1} \times \ell_{1}$ such that neither $A$ nor $A_{12}$ is norm attaining, but such that $A_{21}$ is norm attaining.

Proof. Define $A: \ell_{1} \times \ell_{1} \rightarrow \mathbb{K}$ by

$$
A(x, y):=\sum_{n=1}^{\infty} x_{n}\left(\sum_{k=1}^{n} \frac{k}{k+1} y_{k}\right)
$$

Since $|A(x, y)| \leq \sum_{n, k}\left|x_{n}\right|\left|y_{k}\right|$ and $A\left(e_{n}, e_{n}\right)=n /(n+1),\|A\|=1$. It is also easy that $\|A\|$ is not attained. Moreover, we have:

(i) $A_{12}$ is also not norm attaining, although

(ii) $1=A_{21}(z, z)=\left\|A_{21}\right\|$, for any $z \in \ell_{1}^{* *}$ which is a weak-* cluster point of $\left(e_{n}\right)$.

The verification of (ii) is easy: $A_{21}(z, z)=\lim _{k} \lim _{j} A\left(e_{j}, e_{k}\right)$, for an appropriate subnet. Now, for fixed $k, \lim _{j} A\left(e_{j}, e_{k}\right)=k /(k+1)$, and so

$$
\lim _{k} \lim _{j} A\left(e_{j}, e_{k}\right)=1 .
$$

As for (i), suppose that $A_{12}(z, w)=1$ for some $z, w \in B_{\ell_{1}^{* *}}$. Let $\left(y_{\beta}\right)$ be a net in $B_{\ell_{1}}$ which converges weak-* to $w$. We first observe that if $y_{\beta}=\left(y_{k, \beta}\right)_{k=1}^{\infty}$, then for each $k \in \mathbb{N}, y_{k, \beta} \rightarrow 0$ as $\beta \rightarrow \infty$. Indeed, if this were not the case, then there would exist $k_{0}$ and $\delta>0$ such that $\left|y_{k_{0}, \beta}\right|>\delta$ for some subnet. However, for any $x \in B_{\ell_{1}},\left|A_{12}(x, w)\right|=\lim _{\beta}\left|A\left(x, y_{\beta}\right)\right|$, and each $\left|A\left(x, y_{\beta}\right)\right|$ is at most

$$
\begin{gathered}
\sum_{n=1}^{\infty}\left|x_{n}\right|\left(\sum_{k=1}^{n} \frac{k}{k+1}\left|y_{k, \beta}\right|\right) \leq \sum_{n=1}^{\infty}\left|x_{n}\right|\left(\sum_{k=1, k \neq k_{0}}^{\infty}\left|y_{k, \beta}\right|+\frac{k_{0}}{k_{0}+1}\left|y_{k_{0}, \beta}\right|\right) \\
\leq 1-\left|y_{k_{0}, \beta}\right|+\frac{k_{0}}{k_{0}+1}\left|y_{k_{0}, \beta}\right| \leq 1-\delta\left[1-\frac{k_{0}}{k_{0}+1}\right]
\end{gathered}
$$


which is bounded away from 1 . Thus, for each coordinate $k, y_{k, \beta} \rightarrow 0$.

Next, for any $x \in B_{\ell_{1}}$ and any $\delta>0$, there is $n_{0}$ such that $\sum_{n=n_{0}+1}^{\infty}\left|x_{n}\right|<$ $\delta$. Choose $\beta_{0}$ such that for all $\beta \geq \beta_{0}, \sum_{k=1}^{n_{0}} k /(k+1)\left|y_{k, \beta}\right|<\delta$. We see that

$$
\left|A\left(x, y_{\beta}\right)\right| \leq \sum_{n=1}^{n_{0}}\left|x_{n}\right|\left(\sum_{k=1}^{n} \frac{k}{k+1}\left|y_{k, \beta}\right|\right)+\sum_{n=n_{0}+1}^{\infty}\left|x_{n}\right|<2 \delta .
$$

Since $\delta$ was arbitrary, we conclude that $A_{12}(x, w)=\lim _{\beta} A\left(x, y_{\beta}\right)=0$, and since $x$ was arbitrary it must be that $A_{12}(z, w)=0$, which is a contradiction.

\section{References}

[1] Acosta, M. D., On multilinear mappings attaining their norms, Studia Math., 131 (1998), no. 2, 155-165.

[2] Acosta, M. D., Aguirre, F. J. and Payá, R., There is no bilinear Bishop-Phelps theorem, Isr. J. Math., 93 (1996), 221-227.

[3] Arens, R., The adjoint of a bilinear operation, Proc. Amer. Math. Soc., 2 (1951), 839848.

[4] Aron, R. M. and Berner, P., A Hahn Banach extension theorem for analytic mappings, Bull. Soc. Math. France, 106 (1978), 3-24.

[5] Aron, R. M., Finet, C. and Werner, E., Some remarks on norm-attaining $n$-linear forms, In: K. Jarosz (ed.), Proceedings of the Second Conference on Function Spaces, L. N. Pure and Appl. Math. 172 Marcel-Dekker 1995, pp. 19-28.

[6] Bishop, E. and Phelps, R., A proof that every Banach space is subreflexive, Bull. Amer. Math. Soc., 67 (1961), 97-98.

[7] Choi, Y. S., Norm attaining bilinear forms on $L^{1}[0,1]$, J. Math. Anal. Appl., 211 (1997), 295-300.

[8] Choi, Y. S. and Kim, S. G., Norm or numerical radius attaining multilinear mappings and polynomials, J. London Math. Soc., 54, no. 2 (1996), 135-147.

[9] Davie, A. M. and Gamelin, T. W., A theorem on polynomial-star approximation, Proc. Amer. Math. Soc., 106 (1989), 351-356.

[10] Dineen, S., Complex analysis on infinite dimensional spaces, Springer Monogr. Math., Springer-Verlag London, Ltd., London, 1999.

[11] Finet, C. and Payá, R., Norm attaining operators from $L_{1}$ into $L_{\infty}$, Isr. J. Math., 108 (1998), 139-143.

[12] Jiménez Sevilla, M. and Payá, R., Norm attaining multilinear forms and polynomials on preduals of Lorentz sequence spaces, Studia Math., 127 (1998), 99-112.

[13] Lindenstrauss, J., On operators which attain their norm, Isr. J. Math., 1 (1963), 139148. 